\title{
La construction du canal Seine-Nord Europe et son intégration dans le paysage archéologique
}

\section{Gilles Prilaux et Marc Talon}

\section{(2) OpenEdition}

1 Journals

Édition électronique

URL : https://journals.openedition.org/archeopages/466

DOI : 10.4000/archeopages.466

ISSN : 2269-9872

\section{Éditeur}

INRAP - Institut national de recherches archéologiques préventives

\section{Édition imprimée}

Date de publication : 1 janvier 2012

Pagination : $57-68$

ISSN : $1622-8545$

\section{Référence électronique}

Gilles Prilaux et Marc Talon, « La construction du canal Seine-Nord Europe et son intégration dans le paysage archéologique », Archéopages [En ligne], Hors-série 3 | 2012, mis en ligne le 01 janvier 2012, consulté le 27 février 2023. URL : http://journals.openedition.org/archeopages/466 ; DOI : https:// doi.org/10.4000/archeopages.466 

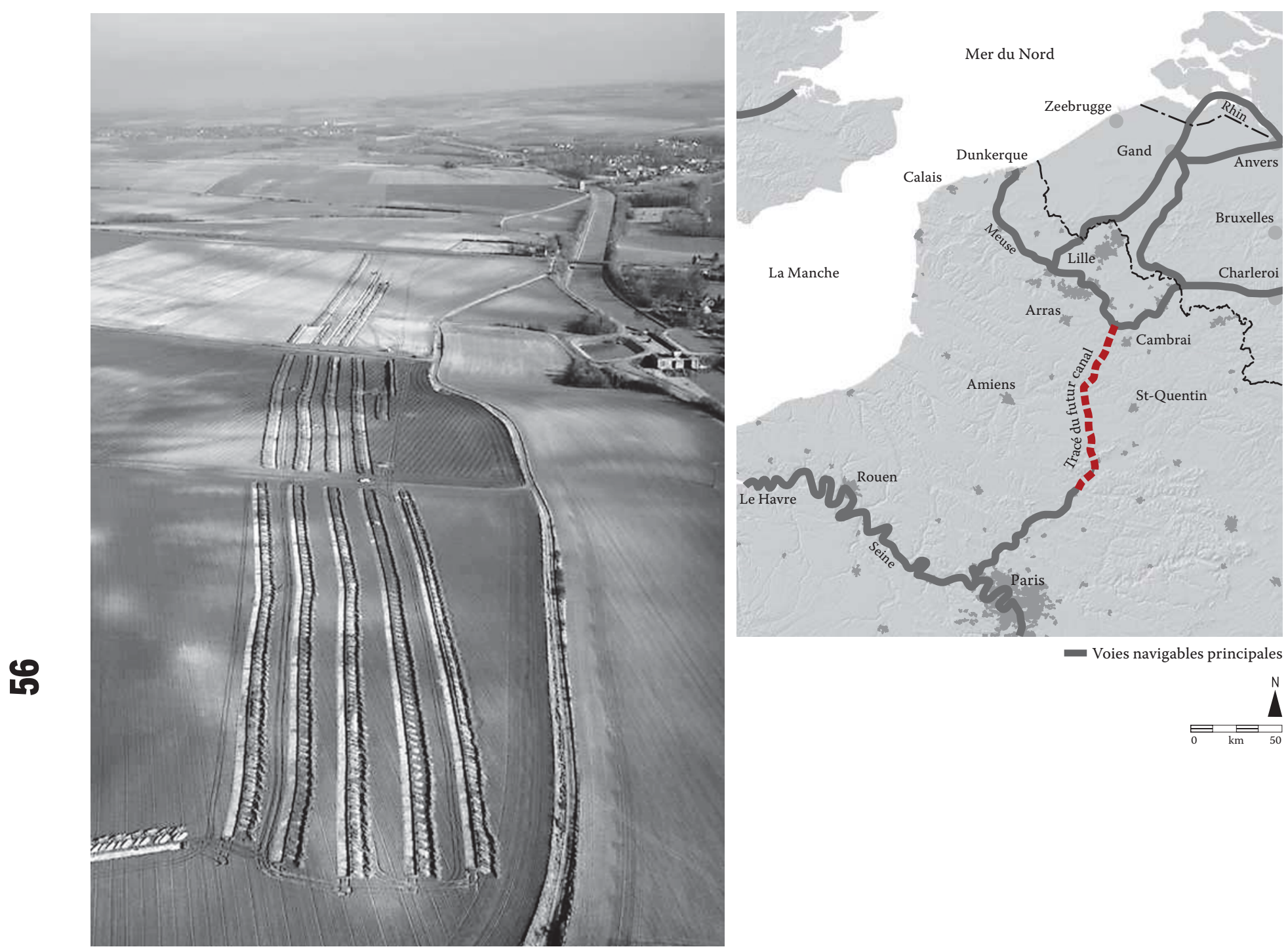

[Fig.1] Vue aérienne des tranchées de sondage en cours sur le tracé du canal Seine-Nord Europe dans le secteur d'Allaines-Moislains (Somme) Diagnostic V. Harnay.
[Fig 2] Carte du tracé du canal Seine Nord Europe en Picardie et Nord-Pas-de-Calais. 
La construction du canal SeineNord Europe et son intégration dans le paysage archéologique

\author{
Gilles Prilaux \\ Inrap \\ Marc Talon \\ Inrap
}

L e programme archéologique du canal SeineNord Europe est un projet ambitieux, identifié comme tel tant par Voies Navigables de France (VNF), maître d'ouvrage, que par les élus, la communauté scientifique, la presse et le grand public. Ce constat tient à la nature de cet ouvrage atypique exceptionnel, aux moyens mis en œuvre par l'Inrap [Fig.1], mais également à l'intérêt que lui ont porté, dès qu'ils en ont eu connaissance, les agents de l'interrégion Nord-Picardie. Ce dernier point, au moment de faire le bilan sur les acquis de l'Inrap, après 10 années d'existence, nous a paru intéressant à développer car, si le programme archéologique du canal Seine-Nord Europe est une bonne vitrine du savoir-faire et des compétences de l'institut, il est également le fruit des expériences et acquis de ses personnels. Sans ce socle, il n'aurait pas pu être élaboré de la même manière et, demain, les résultats de ce programme seront, grâce à cette synergie, intégrés dans des problématiques régionales qu'ils permettront de compléter et renouveler.

Sans revenir sur le caractère gigantesque de l'ouvrage, on peut rappeler que le canal Seine-Nord Europe est destiné à désenclaver le bassin fluvial de la Seine en le reliant au réseau nord-européen. C'est ainsi que les grands ports maritimes comme le Havre, Rouen, Dunkerque, Zeebrugge, Anvers et Rotterdam seront raccordés avec tous les enjeux économiques et environnementaux qui en découleront. Prévu entre Compiègne et Aubencheulau-Bac [Fig.2], l'ouvrage permettra la circulation de barges et de péniches à grand gabarit de la Seine à l'Escaut en traversant 66 communes de l'Oise, de la Somme, du Nord et du Pas-de-Calais. Long de $106 \mathrm{~km}$, le canal achevé aura une largeur de $54 \mathrm{~m}$ et un tirant d'eau de $4,50 \mathrm{~m}$; il comportera 7 écluses, 3 ponts-canaux, 59 ponts routiers et ferroviaires, mais les travaux qu'il implique concernent une emprise de près de 2500 hectares, en moyenne 25 hectares au kilomètre, soit trois fois l'emprise d'une autoroute.

L'histoire du projet archéologique du canal Seine-Nord Europe. Le tracé du canal Seine-Nord Europe recoupe, sur un axe nord-sud, le centre de l'interrégion de l'Inrap pour le secteur de NordPicardie, trois-quarts de cette zone touchant la Picardie et un quart le Nord-Pas-de-Calais. À partir de 2004, les archéologues ont pris progressivement conscience de l'emprise du projet, et ont rapidement mesuré l'intérêt de tels travaux dont l'ampleur est sans commune mesure avec les aménagements réalisés jusqu'alors en France. Certains d'entre eux ont suivi des réunions publiques, d'autres se sont informés auprès des services instructeurs et rapidement le projet, appelé canal Seine-Nord Europe, devint un sujet récurrent de préoccupation pour les archéologues de terrain. La direction interrégionale de Nord-Picardie organisait en juin 2005 une réunion où a été proposé, dans le cadre des réflexions menées sur la mise en place d'axes de recherche, un programme portant sur une démarche d'archéologie préventive raisonnée sur le projet Seine-Nord. Ce thème, qui n'a pas été retenu, illustre toutefois la mobilisation des agents de l'Inrap avant même l'arrivée de la moindre prescription archéologique. Du côté des services de l'État en charge du dossier archéologique, au sein des DRAC, l'intérêt fut tout aussi important et la nomination de Didier Bayard, conservateur du patrimoine au SRA de Picardie comme coordinateur scientifique s'est avéré un signal fort sur la détermination du préfet de région Picardie à soutenir la mise en place d'un programme archéologique à la hauteur de ce projet d'aménagement.

L'instruction du dossier archéologique par les services de l'État se fit parallèlement au lancement, par Voies Navigable de France, d'une étude documentaire et géo-archéologique (2005) par le service archéologique de l'Office National des Forêts, étude qui devait précéder une campagne de prospection pédestre de l'Inrap, intervention finalement jamais réalisée.

Les délais prévus pour l'intervention archéologique ont évolué depuis les premières négociations : en 2006, ils étaient alors très courts, VNF souhaitant alors libérer de toute « contrainte » archéologique le futur partenaire public-privé chargé de la construction puis de la concession de cet équipement. Le transfert devait se faire alors en janvier 2010, date limite donnée par VNF pour la fin des opérations archéologiques (phase «terrain»). Dans un tel dispositif, les contraintes de l'Inrap étaient liées à la trilogie habituelle dans ce type de situation, à savoir le budget, le calendrier et les effectifs. Pour les diagnostics, la contrainte principale, au début du projet, était la conjugaison entre charge de travail et délais très serrés. En effet, la phase de diagnostic portait alors sur près de 2500 hectares, soit $16 \%$ des 15600 ha prescrits sur toute la France en 2006.

Sur la base d'un ratio moyen de 6 journéeshomme $(\mathrm{JH})$ à l'hectare, le total nécessaire s'élevait à environ 15000 journées homme, à répartir initialement sur 18 mois. Pour rappel, le budget total alloué aux diagnostics, sur tout le territoire, était de $70000 \mathrm{JH}$ en 2007, celui attribué à l'interrégion Nord-Picardie, de $8200 \mathrm{JH}$.

Dans la réalité, le programme archéologique commença véritablement en 2008 , sous la coordination de Richard Rougier, adjoint scientifique et technique en Picardie, en attendant la nomination d'un directeur de projet qui fut faite en avril 2009. Mais ce n'est qu'en septembre 2008, une fois la déclaration d'utilité publique (DUP) signée, que les diagnostics débutèrent sur les zones prioritaires correspondant notamment aux emplacements des écluses et autres ouvrages d'art.

Du fait de retard pris dans l'évolution du projet côté VNF, le calendrier des interventions de diagnostic, puis des fouilles, a été modifié de façon importante. Â ce jour, 1700 hectares ont été diagnostiqués et le reste devrait l'être à partir de l'automne 2012. 

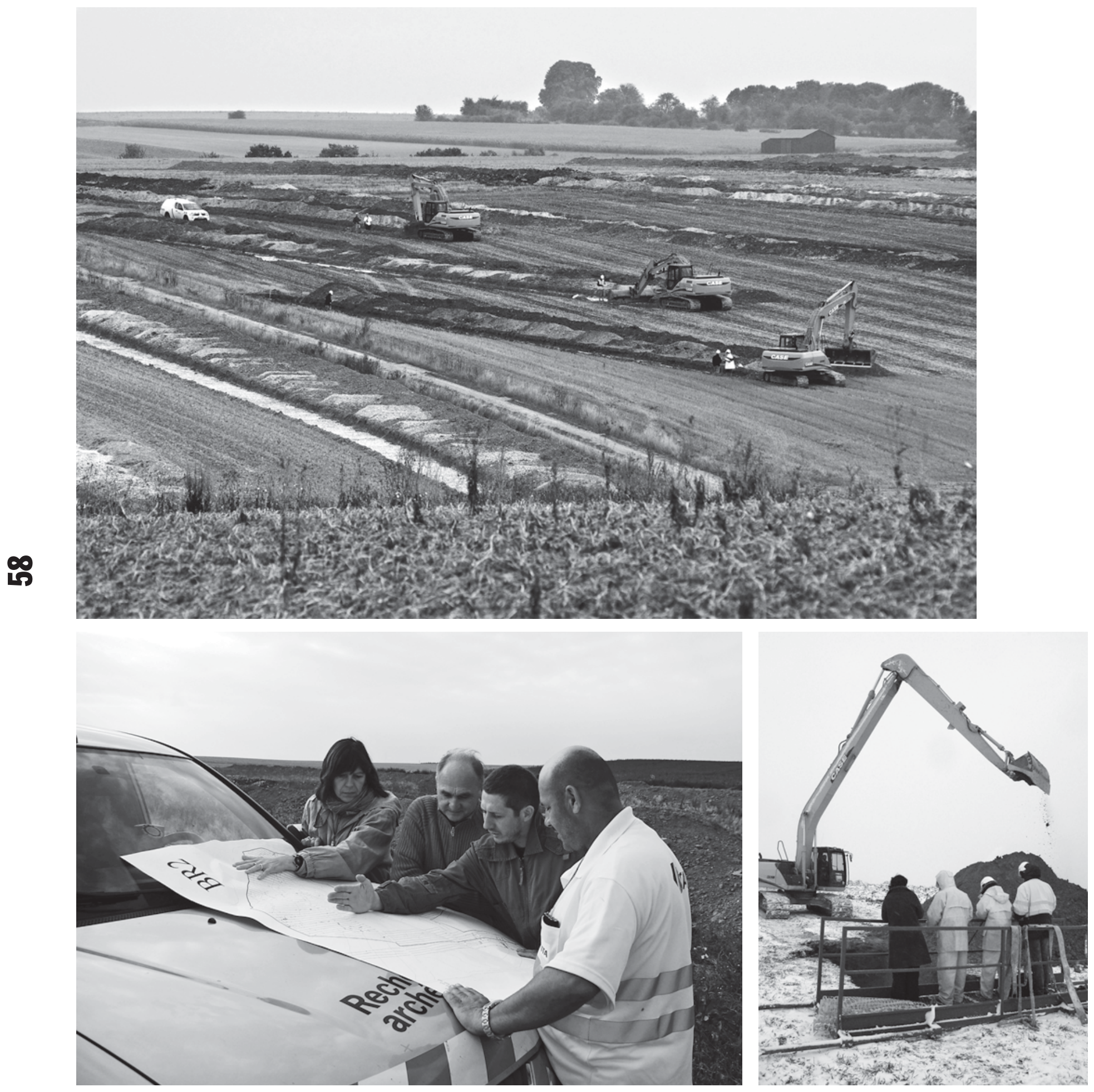

[Fig.3] Tranchées de sondage en cours à l'emplacement du bassin-réservoir du Tarteron à Étricourt-Manancourt

(Somme), ouvrage annexe prévu

sur plus de 80 hectares. Diagnostic Ph. Lefèvre.
[Fig.4] Le contrôle et le suivi des diagnostics sont effectués hebdomadairement par les agents du SRA qui effectuent des visites régulières auprès de chaque équipe.
[Fig.5] Sondage en puits effectué avec une pelle à bras rallongé permettant l'expertise sur un grande profondeur des potentiels niveaux

archéologiques contenus dans les loess, les agents étant en sécurité sur un balcon passerelle mis au point pour ce type d'intervention. Diagnostic E. Goval. 
Tant que le tracé n'a pas été diagnostiqué, il est difficile d'estimer le nombre et le type de sites attendus. Toutefois, la comparaison locale sur les opérations de grands travaux est de un site découvert pour 1o ha sondés. Prenant ce ratio, une prospective a pu être établie en s'appuyant sur une base de 250 sites sur les 2500 ha d'emprise. Mais, les choix faits par le prescripteur, après avis de la commission interrégionale de le recherche archéologique (CIRA), portent sur les sites les plus denses, les plus étendus, les mieux conservés ou encore sur ceux présentant des caractéristiques peu connues (datation, nature, fréquence...). Du fait de ces choix, le nombre de fouilles sera réduit à une centaine d'interventions. Leurs lancements étaient prévu initialement mi-2008 sur 18 mois. Dans les faits, le décalage du calendrier a abouti, d'une part, à la réalisation d'un premier chantier à partir de mars 2010 et, d'autre part, à un étalement des fouilles jusque fin 2012, pour ce qui relève des chantiers sous maittrise d'ouvrage de VNF. À ce planning de terrain, il convient d'ajouter le temps de post-fouilles et d'études, qui sont réalisées pour partie pendant les périodes hivernales (afin de donner une disponibilité maximale des équipes aux phases terrains) et, pour le reste, à l'issue de celles-ci, soit jusqu'en 2014-2015.

\section{Les acquis et compétences des équipes}

de l'Inrap en Nord-Picardie. L'interrégion NordPicardie, dont la surface n'est pas très importante, présente, du fait de sa position au centre du bassin économique européen, une activité conséquente en termes d'aménagement du territoire, activité qui a été accompagnée, depuis plus d'une vingtaine d'années, par des investigations archéologiques (Collart, Talon, 2011).

La professionnalisation de l'archéologie préventive s'est faite progressivement dès les années 1980 puis 1990 (Talon, 2008), à partir des programmes et opérations liés à l'aménagement des centres urbains, l'exploitation de carrières dans les vallées de l'Aisne (Dubouloz, 2008) et de l'Oise (Malrain, 2008), des linéaires autoroutiers (Bayard et al., 2011), du TGV Nord (Saint-Blanquat, 1992), des ZAC et des vastes projets surfaciques (Blancquaert, 2006), l'expérience et les compétences des agents se sont forgées, en grande partie, sur ces grands travaux.

L'activité a été consolidée par le vote de la loi sur l'archéologie préventive en janvier 2001 et la création de l'Inrap. Depuis la fin des années 1980, elle a pu être menée grâce à une synergie entre les représentants des services régionaux de l'archéologie et les agents de l'Afan, puis de l'Inrap, à partir de laquelle un consensus s'est établi sur les modalités de réalisation d'un nombre important de diagnostics. Depuis 2002, en moyenne chaque année sur le secteur de Nord-Picardie, plus d'un millier d'hectares est diagnostiqué, réparti sur 200-250 opérations, générant 25 à 30 fouilles. La nature diversifiée des aménagements et leur contexte topographique ont permis la constitution, pour l'ensemble des périodes depuis la Préhistoire jusquà nos jours, d'un référentiel conséquent tant pour les connaissances archéologiques et paléoenvironnementales que pour les compétences techniques. Ce référentiel a servi de socle pour lélaboration du programme archéologique du canal Seine-Nord Europe qui pourra, à son tour, nourrir les différents thèmes et projets de recherche existants ou à développer.

La composition de l'équipe des agents de l'interrégion constituée actuellement de 180 personnes, avec une production soutenue de publications tant au niveau national qu'international, explique pour partie ces résultats. Leur adhésion aux unités mixtes de recherche associant le CNRS, l'université, le ministère de la Culture et l'Inrap (UMR 8164 «Histoire, Archéologie, Littérature des Mondes Anciens-Institut de Papyrologie et d'Égyptologie de Lille» à Lille 3, UMR 7041 «Archéologie et Sciences de l'Antiquité» à Nanterre, UMR 8591 «Laboratoire de géographie physique» de Meudon et UMR 5197 « Archéozoologie, histoire des sociétés humaines et des peuplements animaux» au Museum National d'Histoire Naturelle de Paris), et leur participation à l'ACR sur les tracés linéaires en Picardie, démontrent ce dynamisme et leur intégration dans la communauté scientifique, quelques-uns d'entre eux siégeant par ailleurs au sein d'instances interrégionales (CIRA) ou nationales (conseil scientifique de l'Inrap, Comité National de la Recherche Archéologique, comité de l'archéologie du CNRS...).

Mais d'autres éléments complètent les atouts de cette interrégion :

- le vivier qu'a pu être le programme «sablières des vallées de l'Aisne et de l'Oise» qui a servi de chantiers-écoles pendant de nombreuses années, les principales périodes concernées par ces fouilles relevant du Néolithique et de la Protohistoire ; - les rencontres faites et les compétences acquises sur les tracés linéaires où ont notamment été développés les savoir-faire en sondages profonds pour la Préhistoire ancienne (Depaepe et al., 2005), les pratiques en archéologie funéraire ou les méthodes de prélèvement pour les études paléoenvironnementales (Talon, 1999);

- les grands travaux surfaciques effectués à partir de la fin des années 1990 -Toyota près de Valenciennes (Cattedu et Roger 2002), Delta 3 à Dourges (Blancquaert, 2007), Actiparc près d'Arras (Jacques et Prilaux, 2003), parc de la Haute Borne à Villeneuve d'Ascq (Quérel, 2003), plate-forme aéro-industrielle de Méaulte (Duvette, Rougier, 2007), parc des autoroutes A26/A29 à Saint-Quentin (Collart et al., 2011) - et liés à l'aménagement, sur des temps très courts, de vastes zones d'activités où ont pu être poursuivis, dans des contextes de plateaux, les premiers acquis d'une archéologie spatiale élaborée dans le cadre des fouilles sur les plaines alluviales;

- la taille raisonnable de ce territoire, où la plupart des archéologues se connaissent, ont la possibilité de se rencontrer dans les journées archéologiques organisées chaque année et de prendre connaissance des travaux effectués dans les bilans scientifiques régionaux (BSR) encore annuellement publiés.

Conscient de l'importance de ces acquis et de la nécessité d'un faire un bilan chiffré et critique, 

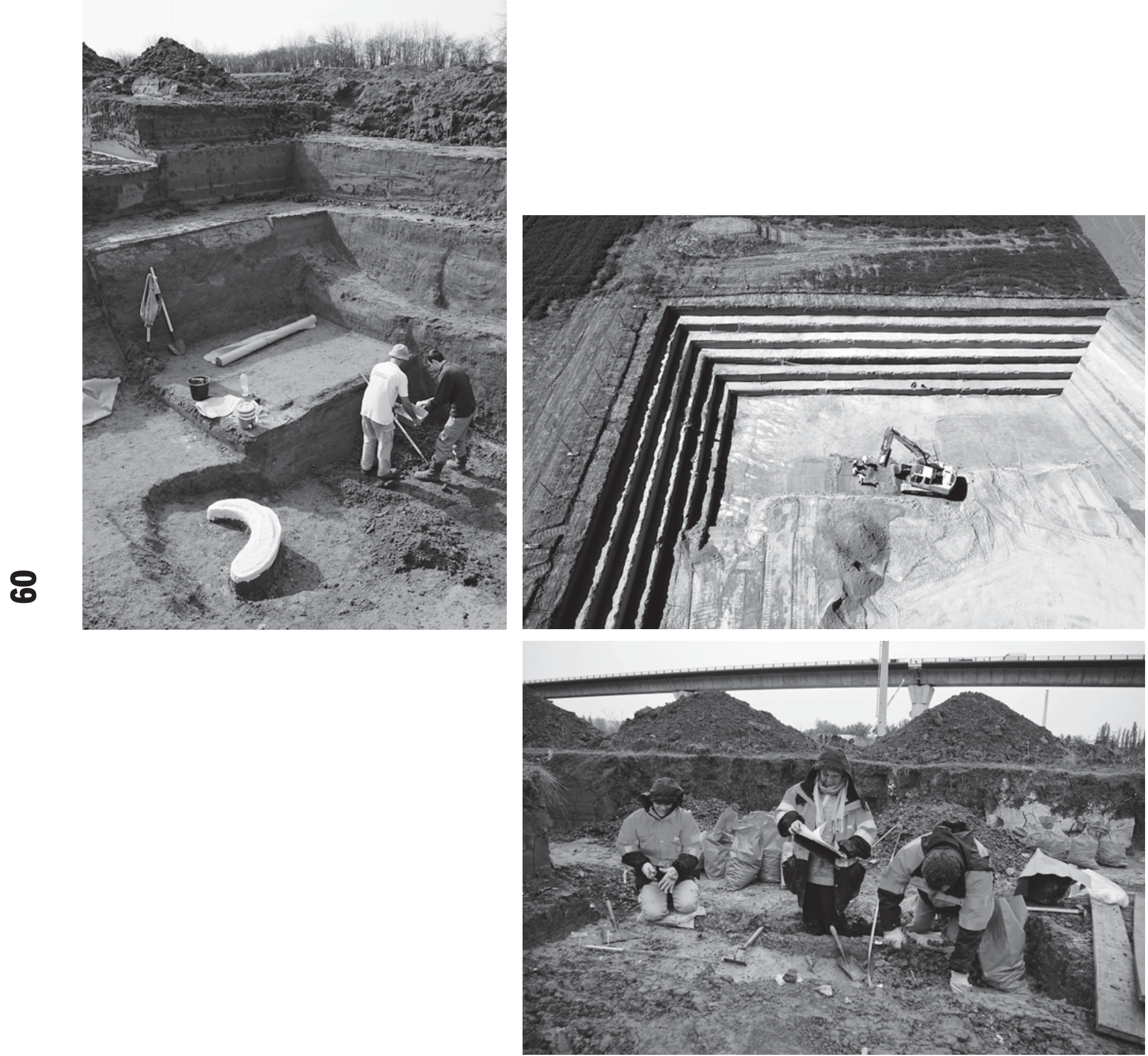

[Fig.6] Une fois un niveau archéologique repéré dans les lœss, il en est réalisé une évaluation en élargissant le sondage afin de pouvoir accéder en toute sécurité aux vestiges. Ici sur la commune de Catigny (Oise), à

environ 4 mètres de profondeur, une défense de mammouth du

Paléolithique moyen a été mise au jour puis protégée avec une gangue de plâtre afin de faciliter son prélèvement. Diagnostic E. Goval.
[Fig 7] La fouille de niveaux de Paléolithique moyen à Havrincourt (Pas-de-Calais) a été prescrite sur une surface de $4500 \mathrm{~m}^{2} \ldots$ à 6 mètres de profondeur. Elle a nécessité la réalisation d'un important chantier de terrassement et d'une succession de gradins permettant l'ouverture d'une fenêtre conséquente sur des occupations qui ont livré du lithique et de la faune datés de 50.000 ans. Fouille E. Goval.
[Fig 8] L'expertise de niveaux mésolithiques préservés dans les limons de débordement de la vallée de l'Oise nécessite le recours à des prélèvements systématiques par quart de mètre carré pour évaluer l'état de conservation des vestiges et leur densité. Diagnostic à Choisy-auBac (Oise) de F. Joseph. 
la communauté archéologique picarde s'est organisée en 2004 pour effectuer, par grandes périodes, un bilan régional dont les différentes synthèses ont été présentées et discutées lors de journées de restitution organisées en Picardie. Ces exposés, effectués par les représentants de l'ensemble des institutions intervenant dans la région, ont été publiés dans la Revue Archéologique de Picardie (Collectif, 2005), support naturel et important de publications pour l'ensemble de la communauté archéologique avec une moyenne d'un millier de pages publiées chaque année.

Larchéologie en Nord-Pas-de-Calais a une histoire différente du fait de l'absence, par exemple, de programme «sablières », mais bénéficie de la présence, plus conséquente qu'en Picardie, de services de collectivités territoriales tournés principalement vers l'archéologie urbaine et les périodes historiques. Du fait de sa position territoriale, elle est le partenaire naturel de programmes européens «Interreg» (Planarch I et II, Boat 1550 BC) auxquels l'Inrap a participé régulièrement, permettant d'y présenter les méthodes et acquis de l'archéologie préventive.

Le canal Seine-Nord-Europe a un impact singulier sur l'archéologie préventive. Pour la reconnaissance des sites archéologiques, il a l'avantage de lier: - du linéaire, permettant un transect d'une région; - du surfacique, découvrant un espace suffisamment étendu en largeur pour avoir une vue d'ensemble de sites;

- de la profondeur, via la découverte de sites préhistoriques enfouis à plusieurs mètres sous la terre arable.

Au regard du bilan de la recherche archéologique régionale et en fonction de la nature des sites et des différentes périodes chronologiques représentées, trois types de fouilles sont attendus:

- la fouille de sites sur de vastes étendues;

- la fouille de sites paléolithiques conservés en profondeur dans les lœss;

- la fouille de sites particulièrement bien conservés dans les fonds de vallons.

La fouille sur de vastes étendues. Du fait de la largeur de l'emprise du canal et des différents ouvrages annexes, des décapages importants sont prévus, en particulier sur les plateaux qui accueillent les plates-formes portuaires multimodales.

Les grandes étendues permettront d'appréhender, pour toutes les périodes, des sites dans leur ensemble, faisant la force et la problématique majeure des fouilles d'aujourd'hui (Brun et al., 2006). L'expérience de Nord-Picardie est importante dans ce domaine et a permis, grâce notamment aux opérations menées sur les sablières et les ZAC, de mettre au point des méthodes d'expertise et d'étude qui ont contribué de façon déterminante à la notion « d'archéologie des réseaux locaux ». L'exploration de centaines d'hectares, soit sur quelques mois, soit dans le cadre du cumul d'interventions archéologiques pendant plusieurs années (Malrain, Pinard, 2006), permettent d'étudier les occupations contemporaines ou diachroniques et de tenter de percevoir le « réseau » d'occupation du sol : généralement les sites ne sont pas de taille similaire et ne sont que partiellement contemporains. Il convient alors d'examiner s'ils ont rempli ou non les mêmes fonctions et s'ils relèvent ou non d'une même catégorie sociale. Par cette vision élargie, les questionnements dépassent le cadre de l'emprise d'un site pris isolément pour porter sur un ensemble de sites, et pour comprendre comment étaient mises en valeur ces occupations à l'échelle d'un terroir. Cette démonstration conduit aujourd'hui à l'élargissement de la notion de site à celle du terroir et à l'appréhension d'espaces géographiques sur la longue durée. Ces sites sont généralement peu enfouis et les vestiges peu denses. Quelles que soient les périodes, la fouille de ces sites s'avère relativement rapide, au regard du nombre de structures attendues. Ce type de site sub-affleurant en contexte de plateau est dégagé mécaniquement et les structures latentes se fouillent à l'aide de moyens mécaniques appropriés, combinés à une fouille manuelle en cas de concentration importante.

Leur détection sous la terre végétale, à 30-50 cm de profondeur, est faite lors des campagnes de sondages systématiques réalisés sur l'ensemble du tracé, méthode éprouvée depuis de nombreuses années. Elle consiste à réaliser de longues tranchées espacées d'une vingtaine de mètres en moyenne, complétées par des extensions de décapage à l'emplacement des concentrations de vestiges. Cette méthode permet de sonder 10\% de la surface des emprises du canal et de ses aménagements annexes [Fig.3]. Sur le canal, compte tenu de l'ampleur des travaux de diagnostic à conduire, ces sondages surfaciques sont réalisés par des échelons opérationnels constitués de 3 à 4 pelles hydrauliques de 180 CV équipées de godets lisses de 3 mètres de large. Ces équipes sont placées sous la direction d'un responsable d'opération assisté de deux techniciens par machine. Le rythme de progression est d'environ un hectare par jour et par engin. Les tranchées de sondages sont rebouchées, au fur et à mesure, après le relevé et l'expertise des vestiges archéologiques découverts et à l'issue du contrôle des agents du SRA [Fig.4].

La fouille de sites paléolithiques dans les loss. Le second type de fouille tient à la nature même des travaux d'aménagement du canal, qui recoupent la couverture lossique du Nord de la France, contexte favorable à la découverte de sites préhistoriques dont les vestiges sont scellés par ces limons fins déposés par le vent aux périodes glaciaires. Les travaux menés lors du creusement du canal du Nord à Noyon en 1913 avaient permis la mise au jour de niveaux paléolithiques constitués de silex taillés et de nombreux restes de faune, assemblage exceptionnel du fait de la présence d'ossements qui, en général, ne se conservent pas dans les limons souvent acides.

Mais dans un premier temps, et cela a été l'un des défis majeurs pour l'équipe archéologique du canal SNE, il a fallu mettre au point une méthode rapide et sécurisée de diagnostic afin de pouvoir repérer, à des profondeurs conséquentes, les éventuels niveaux d'occupation en place. 


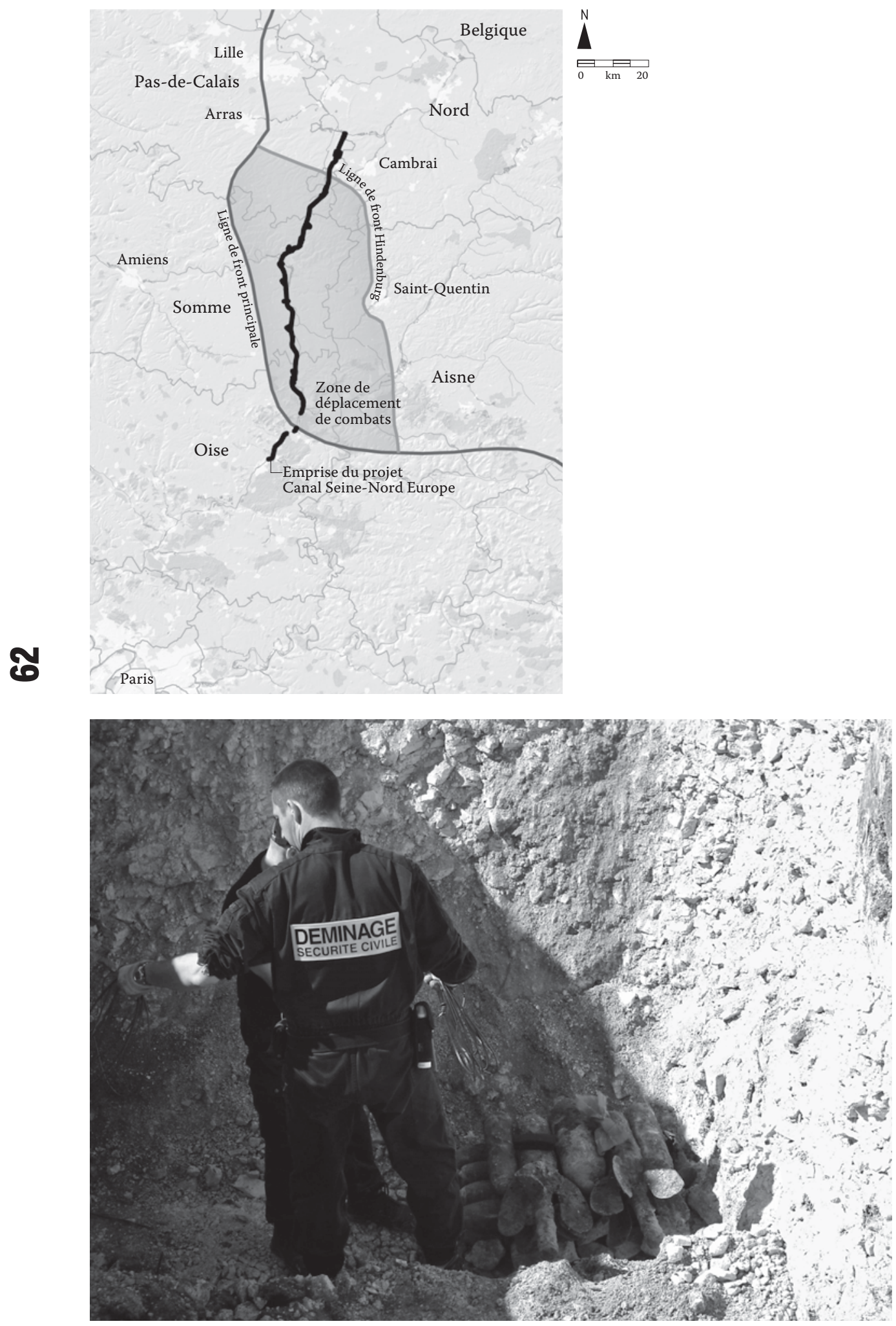

[Fig.9] Carte du tracé du canal SeineNord Europe recoupant les lignes de front de la guerre $14-18$

[Fig.10] Intervention des agents du service de déminage de la Sécurité Civile sur un dépôt de munitions de 120 obus mis au jour sur le bassinréservoir de la Louette à Allaines (Somme). À cause de la sensibilité de ces munitions, leur destruction a été réalisée sur place, après mise en sécurité du secteur. Diagnostic V. Harnay. 
La particularité du canal est en effet d'être un aménagement linéaire qui est quasi essentiellement en décaissement du fait de la nature même de la voie d'eau qui doit être plane entre deux biefs. Cela génère des creusements importants pouvant atteindre jusqu'à 35 mètres de profondeur dans le Pas-de-Calais, sur le secteur d'Hermies.

Depuis plus d'une vingtaine d'années, des compétences ont été développées en Nord Picardie, en collaboration avec le laboratoire de géographie physique de Meudon, dans l'étude de la chronostratigraphie des lœess et le repérage des potentiels niveaux archéologiques conservés (Locht, 2005). Mais l'opportunité de sondages systématiques dans les placages lœssiques sur près de $80 \mathrm{~km}$ de plateaux est exceptionnel à l'échelle européenne, aussi a-t-il fallu développer nos méthodes de manière à optimiser le travail des équipes spécialisées. L'étude chronostratigraphique de ces lœss dont la puissance peut atteindre plusieurs mètres d'épaisseur nécessite le recours à une pelle hydraulique de $180 \mathrm{CV}$ à bras rallongé surnommée «pelle-girafe», permettant la réalisation de sondages en puits à de grandes profondeurs (13-14 $\mathrm{m})$. L'importance de ces travaux, qui ont rarement été menés à de telles profondeurs, a amené l'Inrap à concevoir un système de sécurité spécifique constitué d'un balcon-passerelle permettant l'observation et le relevé sécurisé de la stratigraphie [Fig.5]. En cas de sondage positif, une évaluation est faite en élargissant, sous la forme d'une pyramide inversée en gradins, le puits, permettant ainsi l'expertise directe du niveau archéologique et la fouille sur une petite surface de celui-ci [Fig.6], étape indispensable pour répondre au cahier des charges du diagnostic.

La fouille de niveaux d'occupations paléolithiques à des profondeurs moyennes de 4 à 6 mètres est un exercice auquel les équipes de Nord Picardie sont habituées et leur exploitation scientifique a donné lieu à plusieurs publications qui font aujourd'hui référence notamment dans le domaine de l'analyse comportementale des néandertaliens (Depaepe, 2009). Elle nécessite d'importants terrassements [Fig.7], la mise en sécurité du chantier et l'affectation d'un nombre conséquent d'agents formés à l'exploitation de niveaux paléolithiques pouvant contenir notamment de la faune, élément qui peut rendre l'intervention longue et complexe.

La fouille stratifiée. Le troisième type de fouille est lié à la potentielle préservation de sites pour lesquels une stratigraphie existe, dans les fonds de vallon et dans le lit majeur de la vallée de l'Oise, sachant qu'il n'est pas exclu que des éléments organiques soient conservés dans ces milieux. La méthodologie est alors plus fine et précise. Les décapages, parfois sous d'autres sites de surface, peuvent être suivis d'une fouille fine, manuelle et stratifiée demandant beaucoup de moyens humains et mécaniques ainsi que la mise en œuvre de méthodes de conservation et d'analyses adaptées.

Pour diagnostiquer ce type de milieu, on utilise une pelle hydraulique de $180 \mathrm{CV}$ dirigée par un responsable d'opération, un à deux techniciens et un géomorphologue. La durée de réalisation de ces sondages dépend de l'existence ou pas de stratigraphie, sachant que, pour la vallée de l'Oise, ceux-ci peuvent être effectués pendant une brève partie de l'année seulement, entre avril et octobre, hors des périodes de crue et de remontée de la nappe phréatique. Cette contrainte justifie un rebouchage rapide de ces sondages, au fur et à mesure de leur réalisation, afin d'éviter leur effondrement. Pour comprendre et expertiser les niveaux et les sites, il est indispensable de mener ces sondages avec un géomorphologue, même si le cadre paléoenvironnemental du secteur et l'évolution de la dynamique du régime de la rivière ont pu être posés lors des premières études géomorphologiques menées en collaboration avec le laboratoire de géographie physique de Meudon (Pastre et al., 1997).

La méthode de fouille de vestiges protohistoriques et néolithiques de niveaux conservés a été mise au point dans la moyenne vallée de l'Oise (Prodéo, 1997) à partir d'expériences et d'échanges faits notamment avec l'équipe de Chalain, au début des années 1990. Il est même déjà prévu de l'améliorer sensiblement en bénéficiant du transfert de compétences qui sera issu de la fouille en cours d'Alizay en Haute Normandie.

Pour les sites mésolithiques [Fig.8], qui ont été découverts en nombre dans la vallée de l'Oise, une fois déterminé l'état de conservation de l'occupation et éliminés les sites à palimpsestes, leur fouille bénéficiera des connaissances acquises (Ducrocq, 2005) depuis de nombreuses années sur les fouilles de la vallée de la Somme, de ses affluents et du Thérain.

Les vestiges de la guerre 14-18. En plus des particularités géomorphologiques et archéologiques des territoires recoupés par le canal et auquel répond le dispositif méthodologique adopté, le projet recoupe, à plusieurs reprises, les zones de combats de la guerre 14-18 [Fig.9], dont les vestiges sont relevés et cartographiés, mais non fouillés pour des raisons de sécurité. En complément des formations qui abordent le risque inhérent à notre activité dans le Nord de la France, où 1/3 des communes sont concernées par des vestiges de la Grande Guerre (Desfossés et al., 2008), un stage de sensibilisation aux munitions de guerre a été mis en place avec la Sécurité Civile, stage suivi par les archéologues, par les conducteurs d'engins et par le personnel administratif afin que toute la chaîne de décision soit informée et réactive à ce type de risque. À la fin de l'année 2011, sur l'ensemble des 1700 hectares diagnostiqués, 130 interventions ont été effectuées par les services de déminage de la Sécurité Civile [Fig.10] qui ont traité sur site plus de 7 tonnes de munitions. Parmi ces interventions, notons à deux reprises celles effectuées sur la plate-forme de Marquion, suite à la combustion d'obus au phosphore qui a nécessité, à chaque fois, l'évacuation du chantier et, la seconde fois, la mobilisation d'une quinzaine de véhicules de pompiers venant des différents centres de secours de la région. 

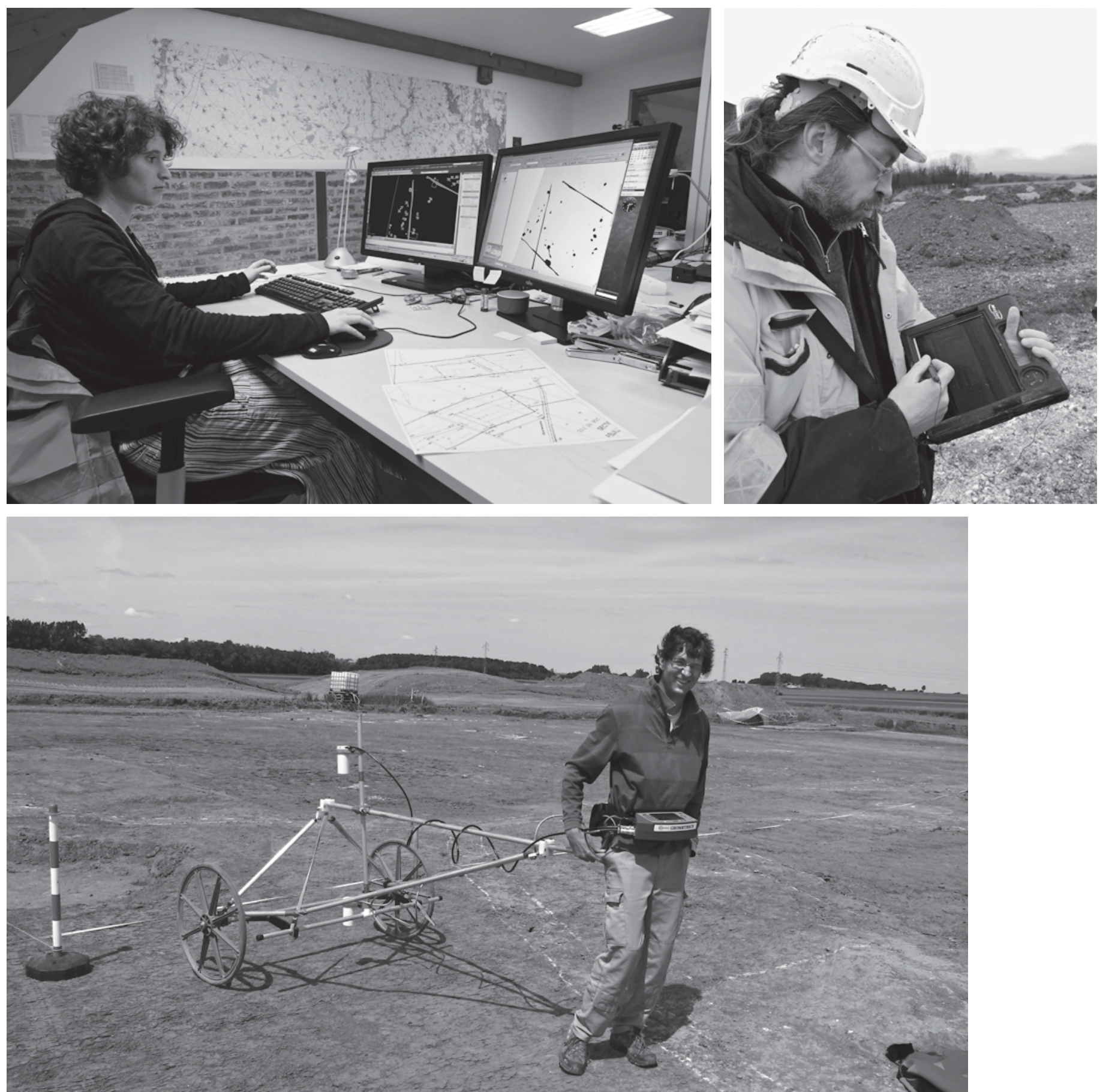

[Fig.11] Afin d'optimiser le traitement du nombre très important de données générées par le programme archéologique du canal Seine-Nord Europe, un SIG a été mis en place dès le début de l'opération, il est géré au sein du plateau technique de CroixMoligneaux par C. Font.
[Fig.12] Les différents types

d'intervention (diagnostic, fouille) sur des substrats et périodes variées, traités dans le cadre d'un plateau technique dédié, représentent une opportunité intéressante pour tester les tablettes PC dans le cadre d'un programme d'expérimentation mis en œuvre par la DST.
[Fig.13] Outils géophysiques mis en œuvre sur site décapé en ayant recours à la mesure de susceptiblité magnétique du sol. Cette technique complète les observations de terrain et G. Hulin en évalue l'efficacité potentielle sur différents types de terrain 
Coordonner et gérer. L'Inrap en tant qu'opérateur désigné pour assurer l'ensemble des diagnostics sur le canal Seine-Nord Europe se trouve ainsi mobilisé depuis 2006 sur cette opération et occupe, depuis avril 2008, des locaux à Croix-Moligneaux, centre archéologique situé au milieu du tracé et consacré à l'opération.

Le projet canal Seine Nord Europe est placé sous la direction d'un directeur de projet rattaché directement à la direction générale. Sa mise en place est accompagnée d'une délégation de signature propre, d'une dotation budgétaire spécifique et d'un accès aux systèmes de gestion opérationnels. Nommé dès la phase de diagnostic, il est prévu que le directeur de projet continuera d'exercer des responsabilités pour les actions postérieures à la réalisation des rapports, intégrant les publications et les restitutions à la communauté scientifique et au grand public.

Pour réaliser sa mission, l'institut est épaulé par une équipe d'une dizaine de personnes, constituée d'un adjoint scientifique (AST), d'un adjoint administratif, d'un contrôleur de gestion, d'une assistante AST et chargée des conventions, d'une chargée de planification, d'une chargée des ressources humaines, d'un assistant tec hnique, d'un conseiller sécurité-prévention, d'un gestionnaire de mobilier archéologique, d'un gestionnaire de centre de ressources, et il bénéficie du soutien de la chargée de communication et de développement culture de Nord Picardie.

Compte tenu du caractère par nature temporaire des opérations liées aux grands travaux, l'affectation des agents CDI s'effectue par application de la procédure des interventions temporaires qui en règle les modalités avec les directions interrégionales concernées. Enfin, pour adapter le fonctionnement des instances spéciales de l'Inrap en restant au plus près de la réalité de terrain, le comité technique paritaire et le comité d'hygiène et sécurité de la direction interrégionale Nord Picardie se tiennent sous la présidence alternée, en fonction des points abordés, du directeur interrégional et du directeur de projet.

Le centre archéologique de Croix-Moligneaux est installé dans une ferme qui a été réhabilitée en fonction des besoins du programme, permettant de créer $1500 \mathrm{~m}^{2}$ de bureaux et d'espaces de travail en capacité d'accueillir soixante-dix agents en post-fouille. Dans le cadre des opérations de diagnostic un parc de véhicules comprenant majoritairement des véhicules $4 \times 4$ ainsi que des roulottes de chantier intégrant réfectoire, vestiaires et sanitaires ont été mis à disposition des équipes, permettant l'accès à tous les types de terrain. Le parc de véhicules ainsi que les matériels de terrassements et les cantonnements de chantier font l'objet de marchés. Le but de ce dispositif est de pouvoir être réactif et organiser les opérations terrain et post-fouilles, de manière à équilibrer la charge d'emploi des personnels et des matériels en fonction des priorités de l'aménageur pour les phases terrain jusqu'à la fin de l'année 2013.

Un effort particulier a été fait pour constituer un pôle technique performant, intégrant la topographie, la réalisation d'un SIG et une chaîne graphique Autocad, éléments permettant notamment la réalisation technique de rapports par la cellule PAO. Ce pôle constitue l'un des maillons importants de l'efficacité de notre dispositif, tant pour les plans produits que pour l'exploitation du SIG [Fig. 11]; il est en relation régulière avec les services de la mission canal SNE de VNF. Le plateau technique travaille en liaison étroite avec les responsables d'opérations, mais devient également centre de ressources pour développer ou tester différentes méthodes et matériels, en collaboration avec la DST et certains spécialistes comme le géophysicien et le coordinateur paléoenvironnement. Le plateau technique du canal SNE est ainsi, de fait, une unité de services (UdS), de déploiement des SIG et d'expérimentation des tablettes PC [Fig. 12]. Dans le même esprit l'utilisation de la susceptibilité magnétique sur site décapé [Fig.13] et la mise en œuvre d'une coordination paléoenvironnentale appliquée à l'ensemble des sites quelle que soit leur datation, leur nature et leur phase d'exploitation (diagnostic, fouilles, études) participent à cette démarche d'innovation.

L'essentiel des agents permanents travaillant sur le projet est issu des effectifs de l'interregion Nord-Picardie. Cet apport est fondamental pour la bonne réalisation des diagnostics et la détection des sites, car les substrats, notamment lossiques, sont de lecture difficile pour des archéologues non habitués. L'expertise est complétée par la collaboration régulière ou ponctuelle avec des spécialistes et référents locaux, permettant une identification du mobilier archéologique et facilitant la mise en contexte des sites. Les équipes de diagnostic, constituées aux 2/3 par des agents permanents, ont été renforcées par quelques personnels sous contrat à durée déterminée (CDD). Pour les fouilles qui nécessitent une augmentation importante des effectifs, le ratio se trouve inversé : à côté de la trentaine d'agents permanents constituée d'encadrants, de spécialistes et de techniciens, plus de quatre-vingts recrutements de CDD ont été effectués. Ils concernent, pour la majorité, des techniciens et une dizaine d'encadrants, formés sur place et bénéficiant d'un tutorat.

Les partenariats. En Nord-Picardie, des partenariats existent avec la quinzaine de services archéologiques de collectivités territoriales agréés. Dans le cadre du canal Seine-Nord Europe, il est prévu de s'appuyer sur ces partenariats pour collaborer à l'exploitation et à la valorisation des fouilles vers le grand public. Dans cet esprit, dès le lancement des premiers diagnostics, l'Inrap a rencontré les responsables de ces services afin d'échanger sur ce principe de partenariat.

Le service archéologique du Douaisis a ainsi mis à disposition, pour les études anthropologiques des sites du nord du tracé, l'une de ses collaboratrices. Pour les sites de l'Arrageois, un suivi scientifique des découvertes du secteur de la plate-forme de Marquion est réalisé avec le service archéologique d'Arras. Un dispositif de valorisation en milieu scolaire a été mis en place avec le service archéologique du Pas-de-Calais et la mission de médiation 


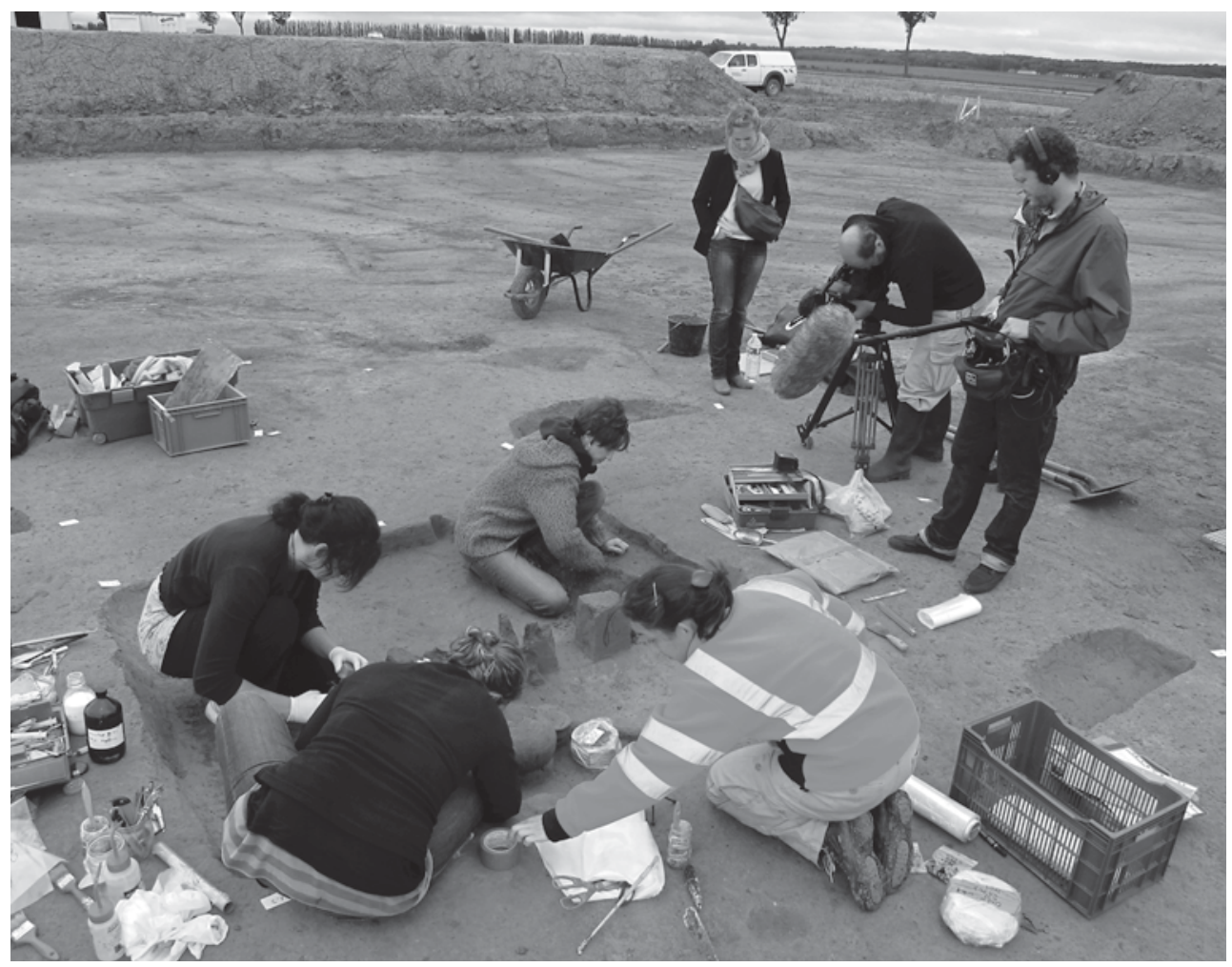

$\varnothing$

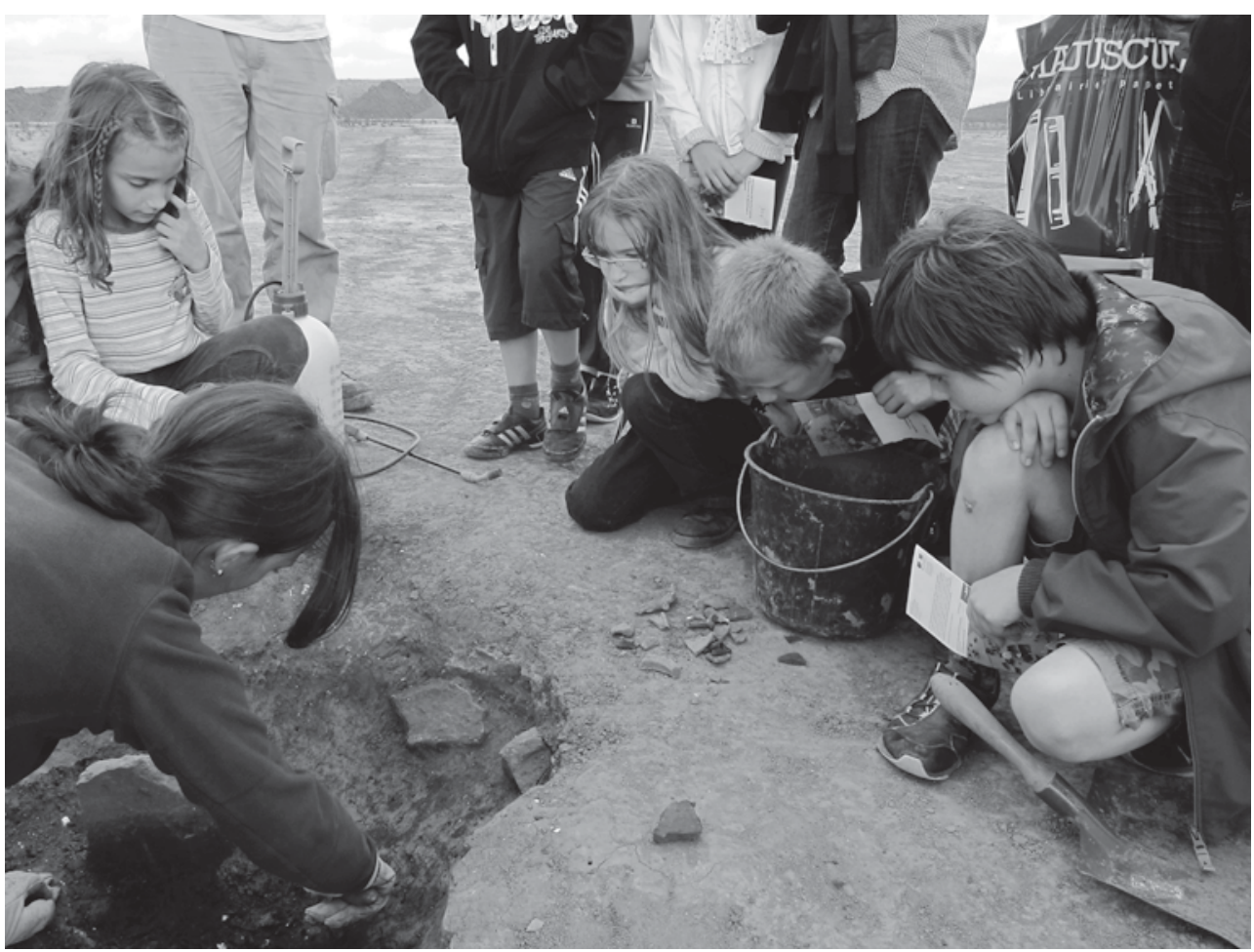

[Fig.14] Démontage en cours d'une sépulture aristocratique gauloise sur le site de la plate-forme de Marquion (Pas-de-Calais) en collaboration avec une équipe de Conservare spécialisée dans le traitement et la conservation des objets métalliques. Cette

découverte importante fait l'objet d'un reportage TV. Fouille N. Soupart.

[Fig.15] Accueil d'une classe de primaire sur le site de la villa gallo-romaine de Marquion. Fouillle D. Gaillard. 
archéologique de l'Inrap par le Conseil Général du Pas-de-Calais

Pour le département de la Somme, bien que son service archéologique ne soit pas agréé, il a été convenu qu'il puisse être mis à contribution en termes de collaboration scientifique, conseil et médiation culturelle. Pour le département de l'Oise, ce jeune service ayant fort à faire sur les travaux d'aménagement du conseil général, il est prévu qu'il puisse collaborer avec l'Inrap sur les sites archéologiques recoupés à la fois par les travaux du canal SNE et les travaux routiers du conseil général. Le département du Nord, bien que peu concerné par les travaux, se situe dans le même état d'esprit. Enfin, le service archéologique de Noyon s'est investi à la hauteur de ses moyens, aux côtés de l'Inrap, son territoire riche de son passé antique étant fortement concerné par les travaux du canal.

À l'occasion des recherches aux archives départementales de la Somme et de l'Oise, l'Inrap a également développé un partenariat fructueux avec ces services qu'elle compte élargir à celui du Pas-de-Calais. Il a permis la numérisation prioritaire de plus d'un millier de plans sur les communes concernées par le tracé du canal.

Les collaborations ont évidemment et naturellement été poursuivies avec les laboratoires installés en Picardie, spécialisés soit dans la détermination et l'analyse de vestiges animaux et végétaux comme, à Compiègne, avec le laboratoire d'archéozoologie et de carpologie du centre de recherche archéologique de la vallée de l'Oise (CRAVO), soit dans la stabilisation et la restauration de mobilier comme, à Soissons, avec le centre d'études des peintures murales romaines (CEPMR) ou, à Compiègne, avec Conservare [Fig.14], qui se consacre au traitement du matériel métallique et céramique.

Enfin, en plus de partenariats nationaux ou interrégionaux déclinés autour du projet du canal, notamment avec les laboratoires du CNRS et de l'université, l'Inrap a mis en place un accord de collaboration avec le département d'Évolution Humaine de l'Institut d'anthropologie évolutive Max Planck à Leipzig.

Communication, médiation, valorisation. Dans le cadre de ses missions d'exploitation des résultats scientifiques et de restitution au public, l'Inrap a mis en place un important dispositif de communication, de médiation et de valorisation en marge des fouilles et pendant la phase post-fouille, voire à l'issue de celle-ci, lorsque l'intérêt des résultats le justifie.

Il concerne tout d'abord linformation des riverains sur les fouilles et leurs enjeux [Fig.15], en partenariat avec l'aménageur, ce qui peut prendre la forme de journées portes ouvertes et de conférences. À cette occasion ou lors de la diffusion régulière de communiqués de presse, des rencontres sont organisées avec les journalistes en fonction des résultats, du contexte et des moyens déployés.

Les échanges avec des partenaires culturels, patrimoniaux et des lieux de culture scientifique et technique régionaux permettent de collaborer sur des actions de restitution comme des expositionsdossiers. Au terme de l'opération archéologique, il est prévu une ambitieuse exposition-bilan en région, dans l'esprit de ce qui a pu être fait avec les expositions «100 00o ans sous les rails » avec Réseau ferré de France, présentée dans 5 musées en région, ou «Aux origines du Loiret» conçu avec Vinci et le département du Loiret. Cette exposition serait accompagnée de la publication d'un ouvrage de synthèse sur les résultats des fouilles.

La production d'un documentaire audiovisuel a également été lancé en collaboration avec VNF, Gédéon Programmes s'en étant vu confié la réalisation.

Enfin, et depuis le printemps 2011, sur le site internet de l'Inrap un atlas interactif est accessible, régulièrement mis à jour et dans lequel sont présentés, au fur et à mesure, les sites fouillés.

La nature du projet du Canal Seine-Nord Europe permet d'articuler les thèmes archéologiques avec des contenus historiques, géographiques, économiques et de les intégrer aux résultats obtenus sur le reste de l'interrégion Nord-Picardie. Cet exercice est facilité par le fait que l'ensemble du dispositif est animé par la chargée de communication et de développement culturel de l'Inrap Nord-Picardie, en concertation avec la direction de la communication et du développement culturel au niveau national.

Pour tout observateur extérieur, le programme archéologique du canal Seine Nord Europe est la réponse adaptée à la taille et à l'importance du potentiel archéologique que cet équipement hors du commun présente. Face aux enjeux de cet ambitieux projet d'aménagement du territoire, il témoigne du grand intérêt de la communauté scientifique de l'interrégion Nord Picardie et du soutien de la direction de l'Inrap. Ce programme archéologique n'est pas une simple réponse faite à une opportunité, mais le résultat de la mobilisation d'agents dont le niveau de compétences et de connaissances est à la mesure des moyens à déployer pour faire face à cet énorme coup de griffe provoqué par la construction d'une nouvelle voie d'eau dans le paysage de Nord-Picardie.

La mise en place d'un tel programme, pour lequel une direction de projet a été créée, s'appuie sur la maturité scientifique, méthodologique et technique des équipes de l'interrégion, fruit d'expériences diverses et conséquentes menées par l'Afan, puis l'Inrap, sur ces territoires. Arrivé, quelques années plus tôt, la prise en charge de ce projet d'aménagement n'aurait pas été de même nature ni de même qualité. Indéniablement le canal Seine-Nord Europe présente l'opportunité d'exposer le savoir-faire et les acquis de l'archéologie préventive, mais aussi les capacités de l'Inrap à continuer à développer les connaissances scientifiques et techniques, indispensables corollaires à l'évolution de notre discipline. 
Bayard D., Buchez N., Depaepe P. (DIR.), 2011 : «Quinze ans d'archéologie préventive sur les tracés linéaires en Picardie», Revue Archéologique de Picardie, $\mathrm{n}^{\circ}$ 3-4.

BLANCQUAERT G., 2006: «Étude comparative de 4 opérations archéologiques réalisées en milieu rural dans la région Nord-Pas-deCalais - France», in Blancquaert G., Medlycott M. (Dir.), Archaeological Evaluation of Rural Areas in the Planarch Area of North West Europe, Planarch 2, Action 2C, Maidstone, p. 19-38.

BLANCQUAERT G. (DIR.), 2007 : «Résultats des fouilles préventives Delta 3 , plate-forme multimodale de Dourges (62)» Gauheria, hors-série, 120 p., 85 fig.

Brun P., MARCigny C., VAnMoerkerke J. (DIr.), 2006 : Une archéologie des réseaux locaux. Quelles surfaces étudier pour quelle représentativité?, Actes de la table ronde de Châlons-en-Champagne, 14-15 juin 2005, Les Nouvelles de l'archéologie, $\mathrm{n}^{\circ}$ 104, $96 \mathrm{p}$.

Cattedu I., Roger D., 2002 : «L'opération Onnaing-Toyota en 2 parties ", Archéologia, n' 385 , p. 48-57, et n 386, p. 42-51.

Collart J.-L., Talon M, 2011 : Fouilles et découvertes en Picardie, éd. Ouest-France et Inrap (Coll. Histoire), 144 p.

Collart J.-L., Hosdez Ch., Lemaire P., 2011 : 350 ooo ans d'Histoire: Découvertes archéologiques dans le Saint-Quentinois, Catalogue d'exposition, Ville de Saint-Quentin, DRAC de Picardie, Inrap, 168 p., 277 fig.

Collectif, 2005 : La Recherche archéologique en Picardie : bilans et perspectives, Revue archéologique de Picardie, no 3-4, 346 p.

Depaepe P., Locht J.-L., Swinnen C., 2005 : « Méthodes de détection de sites du Paléolithique ancien et moyen en archéologie préventive», in Recherches archéologiques préalables à l'aménagement des zones d'activité économique, Actes des journées d'Archéologie en Wallonie 2004, AWPA asbl, Liège, p. 51-59.

Depaepe P., 2009 : La France du Paléolithique, Paris, La Découverte, 180 p.

Desfossés Y., JaCQues A., Prilaux G., 2008 : L'archéologie de la Grande Guerre, éd. Ouest-France (Coll. Histoire) et Inrap, $128 \mathrm{p}$.

Dubouloz J., 2008: «Beaucoup de sable et un peu de terre noire...», Archéopages, hors-série, p. 49-55.

DUCROCQ T., 2005 : «Le Mésolithique », La recherche archéologique en Picardie : bilans et perspectives, Revue archéologique de Picardie, $\mathrm{n}^{\circ}$ 3-4, Amiens, p. 40-49.

Duvette L., Rougier R., 2007 : Méaulte (Somme) : la plate-forme aéro-industrielle de Haute-Picardie, plaquette Archéologie en Picardie, DRAC de Picardie, nº 35, 12 p., 26 fig.

JaCques A., Prilaux G., 2003 : Dans le sillage de César : traces de romanisation d'un territoire, les fouilles d'Actiparc à Arras, Catalogue d'exposition, Musée des Beaux-Arts d'Arras, 2003, Service Archéologique de la ville d'Arras et Inrap, 8 o p.

Locht J.-L., 2005 : «Le Paléolithique moyen en Picardie : état de la recherche ", La recherche archéologique en Picardie : bilans et perspectives, Revue archéologique de Picardie, n 3-4, Amiens, p. 27-35.

Malrain F., PinARD E., 2006 : Les sites laténiens de la moyenne vallée de l'Oise du Ve au Ier s. avant notre ère, Revue Archéologique de Picardie, numéro spécial 23, 268 p., 174 fig.

Malrain F., 2008 : «Silence au fond des vallées », Archéopages, hors série, p. 119-120.

Pastre J.-F., Fontugne M., Kuzucuoglu C., Leroyer C., LimondinLozouet N., TAlon M., Tisnerat N., 1997 : «L'évolution tardiglaciaire et postglaciaire des lits fluviaux au nord-est de Paris (France). Relations avec les données paléoenvironnentales et l'impact anthropique sur les versants », Géomorphologie, 4, p. 291-312.

Prodeo F., 1997 : «Une méthode d'analyse des sites fossilisés en contexte alluvial», in Espaces physiques, espaces sociaux dans l'analyse interne des sites du Néolithique à l'âge du Fer, $119^{\mathrm{e}}$ congrès CTHS, Amiens, 1994, p. 207-232, 13 fig.

QUÉrEL P., 2003 : «Le Parc Scientifique de la Haute Borne à Villeneuve d'Ascq (Nord) », Archéopages, 9, Inrap, p. 6-11.

Saint-Blanquat H. de, 1992 : Archéo TGV, 450 km d'histoire, Paris, Casterman.

TALON M., 1999: «La pratique de l'archéologie environnementale à l'AFAN », in Environnement et archéologie, actes des assises de Nanterre, mai 1999, Les Nouvelles de l'Archéologie, n ${ }^{\circ}$ 78, p. 29-31.

TALON M., 2008 : «Évolution et professionnalisation de l'archéologie préventive en milieu rural dans le nord de la France», Archéopages, hors-série, p. 65-69.

TALON M., Bellan G., 2009 : «Développement et professionnalisation de l'archéologie préventive en France : l'Afan (1973-2001)», in Demoule J.-P., LANDes CH. (DIR.) La fabrique de l'archéologie, Actes du colloque de Paris (Inrap-Inhaa), février 20o8, Paris, La Découverte, p. 251-265. 